\title{
Compreendendo Relações entre Estratégias de Aprendizagem e a Ansiedade de Alunos do Ensino Fundamental de Campinas
}

\author{
Elis Regina da Costa ${ }^{1}$ \\ Evely Boruchovitch \\ Universidade Estadual de Campinas
}

\begin{abstract}
Resumo
Pesquisas sugerem que um bom desempenho escolar envolve o uso eficiente de estratégias de aprendizagem e o controle de variáveis psicológicas do aluno. Tendo como base teórica a Psicologia Cognitiva baseada na Teoria do Processamento da Informação, este estudo verificou relações entre o uso de estratégias de aprendizagem e a ansiedade de 155 alunos do ensino fundamental de uma escola pública de Campinas. Utilizou-se como instrumentos para a coleta de dados uma entrevista estruturada sobre estratégias de aprendizagem e uma escala de ansiedade. De modo geral, os resultados parecem indicar que a ansiedade pode tanto favorecer, quanto interferir no uso adequado de estratégias de aprendizagem. Os dados são discutidos em termos de suas implicações educacionais e direções para futuras pesquisas sobre essa temática são apresentadas.

Palavras-chave: Estratégias de aprendizagem; ansiedade; ensino fundamental.
\end{abstract}

\section{Understanding Relationships Learning Strategies and Elementary School Student's Anxiety of Campinas}

\begin{abstract}
A number of studies suggest that good academic achievement involves both the use of efficient learning strategies and control of the student's psychological variables. Using Cognitive Psychology, more specifically an Information Processing-based approach as a theoretical framework, the present study examined the relations between the use of learning strategy and anxiety of 155 elementary school students of a public school in Campinas. A structured interview on learning strategies and an anxiety scale were used. Overall, results seem to indicate that anxiety can either favor or interfere with the use of learning strategies. Results are discussed in terms of their educational implications. Directions for further research on this topic are also addressed.

Keywords: Learning strategies; anxiety; elementary school.
\end{abstract}

Psicólogos cognitivistas adeptos da perspectiva do processamento da informação enfatizam a importância dos processos psicológicos para explicar o que acontece quando o indivíduo lida com uma tarefa ou um problema (Flavell, P. H. Miller \& S. A. Miller, 1999). Tendo como base a analogia entre a mente humana e o funcionamento de um computador, os psicólogos cognitivistas que defendem essa abordagem passaram a conceber o ser humano como um processador de informações.

O desenvolvimento cognitivo para esses teóricos é entendido em termos de mudanças e diferenças funcionais na capacidade de processamento da informação entre crianças e adultos explicado como conseqüência do desenvolvimento da eficácia operacional das estratégias de aprendizagem utilizadas e pelo conhecimento das tarefas. Esses teóricos acreditam que, no processo de aprendizagem, há a interação entre fatores contextuais e fatores internos do aprendiz. Contudo, privilegiam-se transformações internas, relacionadas a mudanças no uso das estratégias de aprendizagem (Boruchovitch, 1993a).

Segundo Dembo (1994), estratégias de aprendizagem são técnicas ou métodos que os estudantes utilizam para obter e

\footnotetext{
${ }^{1}$ Endereço para correspondência: Rua Colônia de Minas, 500/74A. Santa Amália, Campinas, SP, 13080 970. Fone: (19) 32286172.E-mail:ecosta@unicamp.br
}

aprender uma dada informação. De acordo com Nisbett, Schucksmith e Dansereau (1978, citado em Pozo, 1995), as estratégias de aprendizagem são atividades ou procedimentos utilizados com o propósito de facilitar a aquisição, o armazenamento e/ou a utilização da informação. Podem ainda ser definidas como processos conscientes controlados pelos estudantes para atingirem objetivos de aprendizagem, bem como qualquer procedimento adotado para a realização de uma determinada tarefa (Da Silva \& De Sá, 1997). Uma descrição mais detalhada sobre estratégias de aprendizagem pode ser encontrada em Boruchovitch (1993a, 1999), Dembo (1994) e Woolfolk (2000).

As estratégias de aprendizagem usadas espontaneamente ou adquiridas por meio de intervenções sistemáticas, bem como os aspectos emocionais e afetivos que favorecem ou não o comportamento estratégico, têm sido investigadas recentemente por pesquisadores (Brown, 1997; Corno \& Mandinach, 1983; Purdie \& Hattie, 1996; Zimmerman, 1986; Zimmerman \& Martinez-Pons, 1986). Investigações comprovam a eficiência da instrução em estratégias de aprendizagem, para a melhoria da aprendizagem e do desempenho escolar. Estudos ressaltam que a ansiedade, a motivação, as crenças sobre inteligência, a auto-eficácia, as atribuições de causalidade, dentre outros fatores, influenciam a utilização das estratégias de aprendizagem por parte dos 
alunos. Pesquisas sobre estratégias de aprendizagem e os fatores que interferem no seu uso, no Brasil, são ainda escassas.

\section{Ansiedade: Uma Breve Introdução}

Ajuriaguerra (1976) define a ansiedade como caracterizada por uma "sensação de perigo iminente, aliada a uma atitude de expectativa, que provoca uma perturbação mais ou menos profunda" (p. 607). Schaie e Geiwitz (1982) definem ansiedade como um estado de estímulo caracterizado por um medo vago. Já Drever (1952, citado em Rosamilha, 1971) a descreve como um estado emocional complexo e crônico, onde o componente mais importante é a apreensão ou o receio. $\mathrm{O}$ propósito ou a função da ansiedade é provavelmente facilitar a detecção do perigo ou ameaça em potencial (Eysenck, 1992).

Freud (1894) contribuiu de forma significativa para o desenvolvimento das pesquisas sobre a ansiedade. Até o momento de ser conceituada e discutida por Freud, a ansiedade somente havia sido estudada no campo da filosofia (citado em Rosamilha, 1971). Baseado em fenômenos clínicos observáveis, Freud afirma que ansiedade faz parte do instinto de conservação do indivíduo, e é herdada filogeneticamente. Sua origem estaria ligada ao trauma do nascimento e ao medo da castração (May, 1980). Freud classificou a ansiedade em ansiedade normal e ansiedade neurótica. A primeira relacionase com a consciência de um perigo externo e real, como a morte. A ansiedade neurótica por sua vez ocorreria em situações com ausência de ameaça de perigo real. Ela não seria percebida conscientemente, pois seria reprimida. A partir do momento em que conceituou a ansiedade como patológica, Freud restringiu seu foco de interesse aos efeitos neuróticos da ansiedade (May, 1980; Pessoti, 1977; Rocha, 1976). As concepções teóricas de Freud sobre ansiedade repercutiram nos estudos desenvolvidos posteriormente (May, 1980; Rocha, 1976).

A diferenciação dos componentes da ansiedade é bastante ampla. Eysenck (1992) identificou três sistemas de respostas: a) a comportamental, b) a fisiológica e c) a verbal. Buss (1966, citados em Rosamilha, 1971) distinguiu quatro sistemas compondo a ansiedade. O sistema somático (suor, palpitação cardíaca, ruborização), sistema afetivo (agitação, pânico, irritabilidade), sistema cognitivo (inquietude, distração, esquecimento) e sistema motor (tensão muscular, tremores, calafrios). De forma geral ao se fazer uma análise de tais diferenciações percebe-se que estas podem ser englobadas em dois componentes principais, o cognitivo e o somático.

Spielberger (1966, 1972, citado em Rocha, 1976) propôs uma distinção entre ansiedade estado e ansiedade traço integrando estudos anteriores de Cattell e Scheier (1961), assim como as concepções teóricas de Freud. Essa diferenciação foi altamente relevante para o desenvolvimento de investigações sobre ansiedade. Em seu trabalho, Rocha (1976) descreve como Spielberger $(1966,1972)$ diferencia a ansiedade como estado e ansiedade como traço de personalidade:

Ansiedade estado refere-se a um estado ou condição emocional transitória do organismo humano que varia em intensidade e através do tempo. Caracteriza-se por sentimentos subjetivos, conscientemente percebidos, de apreensão e tensão, e ativação do sistema nervoso autônomo. O nível de ansiedade estado é alto em situações percebidas como ameaçadoras pelo indivíduo, independentemente do perigo objetivo, sua intensidade é menor em situações não tensionadoras.

Ansiedade traço refere-se a diferenças na disposição a perceber uma ampla escala de situações estimuladoras como perigosas ou ameaçadoras e na tendência a responder a tais ameaças com reações de ansiedade estado. As pessoas que possuem alta ansiedade traço tendem a perceber um maior número de situações como perigosas, ou ameaçadoras do que as de baixa ansiedade traço e a responder situações ameaçadoras com elevação de ansiedade estado. (Rocha, 1976, p. 13)

Cabe destacar que alguns trabalhos teóricos e experimentais sobre ansiedade vêm sendo crescentemente desenvolvidos no Brasil. Como descrito em La Rosa (1998), algumas investigações realizadas associam a ansiedade a diversos tópicos como julgamento moral, depressão, baixo rendimento no vestibular, primeira relação sexual e migração. A ansiedade dos pais e a adaptação de seus filhos à préescola e as relações entre a resposta de ansiedade de pais e a resposta de ansiedade de seus filhos foram também identificadas por Biaggio (1998) como temas que vem sendo estudados. Existem poucos dados empíricos sobre o efeito da ansiedade no desempenho escolar (Bzuneck, 1991; Bzuneck \& Silva, 1989), sobretudo que utilizam como referencial teórico a psicologia cognitiva baseada no processamento da informação. Considerando a importância da ansiedade como variável fundamental da personalidade humana, faz-se necessário que esse tema seja mais amplamente investigado nas mais diferentes direções. Tendo em vista os objetivos do presente estudo, a seguir, uma análise da ansiedade escolar na abordagem da psicologia cognitiva baseada no processamento da informação será apresentada.

\section{A Ansiedade Escolar e a Abordagem da Psicologia Cognitiva Baseada na Teoria do Processamento da Informação}

Estudos realizados nos últimos 30 anos apóiam a hipótese de que a alta ansiedade interfere negativamente na 
performance acadêmica (Monteiro, 1980; Rocha, 1976; Tobias, 1980, 1985; Wigfield \& Eccles, 1989). Nas décadas de 1960 e 1970, a ansiedade escolar foi bastante pesquisada. Após esse período, a maior parte das investigações realizadas no contexto acadêmico tem sido desenvolvida pelos teóricos da Psicologia Cognitiva baseada no Processamento da Informação, que defendem que o uso adequado de estratégias de aprendizagem e a manutenção de um estado interno satisfatório favorecem o desempenho escolar. Esse estado interno satisfatório refere-se ao controle de diversas variáveis, como motivação para aprender, atribuições de causalidade para situações de sucesso e fracasso escolar, controle da ansiedade, entre outras.

A ansiedade é assumida pelos adeptos da abordagem do processamento da informação como sendo um constructo multidimensional, constituído por dois aspectos distintos, mas relacionados: a preocupação e a emotividade. Enquanto a preocupação se refere ao componente cognitivo - ou seja, as expectativas negativas sobre si mesmo, preocupações com as conseqüências potenciais -, a emotividade engloba a parte fisiológica, como sintomas físicos, sentimentos de desprazer, nervosismo e tensão (Tobias, 1980, 1985; Wigfield \& Eccles, 1989).

Segundo Wigfield e Eccles (1989), a ansiedade pode afetar tanto alunos com alto como com baixo desempenho. Alunos bem-sucedidos podem tornar-se ansiosos por causa das expectativas não realistas dos pais, dos colegas ou mesmo suas, de que devem ter um ótimo desempenho em todas as disciplinas. No caso de alunos com baixo desempenho, se as situações de fracasso na escola se repetem, a ansiedade pode aumentar como conseqüência do desempenho pobre.

A ansiedade experimentada pelos alunos na situação de prova tem sido a mais investigada. Duas interpretações complementares e não excludentes tentam explicar o baixo desempenho de alunos ansiosos em provas (Tobias, 1985). O primeiro modelo, denominado de interferência (Geen, 1980; Mueller, 1980; Saranson, 1980, 1986; Wine, 1971, 1980, citados em Wigfield \& Eccles, 1989), defende que a ansiedade em situações de prova acaba por interferir na capacidade de recordar ou recuperar um conteúdo aprendido anteriormente, num momento de tensão. Aventa-se a hipótese de que alunos com alta ansiedade fracassam na situação de inventário, pois dividem sua atenção entre as exigências da tarefa e sentimentos de autodepreciação, diminuindo o nível de concentração e o desempenho em situações estressantes de avaliação. Assume-se que a aprendizagem ocorreu, mas o aluno não é capaz de demonstrar devido à ansiedade causada pela situação de avaliação (Wigfield \& Eccles, 1989).

Já o segundo modelo, chamado de déficit (Benjamin, McKeachie, Lin \& Holinger, 1981) relaciona a ansiedade com hábitos de estudo e estratégias de aprendizagem. Os teóricos do modelo do déficit defendem que o baixo desempenho de alunos altamente ansiosos pode ser explicado por dois aspectos: deficiências nos hábitos de estudo e nas estratégias de aprendizagem utilizadas na preparação para as situações de avaliação. Tais déficits ocorreriam, tanto no momento da aquisição, como no armazenamento do conhecimento. Contudo, evidências parecem indicar que o maior problema dos alunos ansiosos é com a recuperação do conteúdo, e não com o armazenamento (Tobias, 1980, 1985). A ansiedade dos alunos aumentaria devido à consciência de que não dominam o conteúdo e de que não sabem utilizar estratégias de aprendizagem de forma adequada nas situações de avaliação escolar. Pesquisas vêm demonstrando que alunos muito ansiosos possuem hábitos de estudo inadequados quando comparados a outros pouco ansiosos, bem como passam maior quantidade de tempo estudando; é, contudo, a qualidade dessa dedicação mais importante do que a quantidade. De forma geral, alunos com alta ansiedade possuem também um conhecimento deficiente sobre estratégias para preparação a exames.

Uma investigação conduzida por Benjamin, Mckeachie e Lin (1987, citado em Naveh-Benjamin, 1991) forneceu apoio adicional para o modelo do déficit no processamento da informação em relação à ansiedade. O objetivo desta pesquisa foi analisar em situação de sala de aula como os alunos estavam organizando os conteúdos de aprendizagem. Os resultados permitiram verificar que os alunos com alta ansiedade e bons hábitos de estudo conseguiam aprender e ter um bom desempenho na situação em que a avaliação aparecia como um exercício de revisão. Quando a avaliação era uma prova tradicional, continuavam com baixo rendimento, mesmo possuindo bons hábitos de estudo. Já os estudantes com alta ansiedade e hábitos de estudo inadequados apresentaram baixo aproveitamento nas duas situações de avaliação (de exercício e de prova). Esses dados sugerem que a alta ansiedade interfere na aprendizagem (Naveh-Benjamin, 1991).

A hipótese da capacidade de processamento cognitivo limitada foi uma terceira hipótese proposta por Tobias (1985), mostrando-se útil para entender a complementaridade das hipóteses dos modelos anteriormente descritos (do déficit e da interferência). Ao assumir-se que os indivíduos possuem uma capacidade de processamento de informação limitada, considerou-se que o componente cognitivo da ansiedade absorveria parte dessa capacidade e as demandas da tarefa absorveriam outra parte. Isso excederia a capacidade de processamento da informação disponível naquele momento e interferiria na aprendizagem, gerando um decréscimo no desempenho, nas provas. Na realidade, hábitos de estudo e estratégias de realização de provas ineficazes, assim como alta ansiedade nas situações de avaliação, sobrecarregariam 
a capacidade cognitiva disponível. A melhor performance seria encontrada em alunos com hábitos de estudos adequados que utilizam estratégias de aprendizagem e de realização de provas eficientes e não possuem alta ansiedade.

Teóricos que adotaram o referencial da emotividade e da preocupação e o associaram com os componentes de ansiedade traço e estado, observaram que as maiores diferenças entre os alunos com alta e baixa ansiedade não é o nível de estimulação fisiológica durante os inventários, mas a reação cognitiva, que pode ser de facilitação ou de debilitação (Morris, Davis \& Hutchings, 1981).

Tendo em vista que um bom desempenho escolar envolve o uso eficiente de estratégias de aprendizagem e o controle de variáveis psicológicas do aluno, esta investigação teve como objetivo verificar relações entre o uso de estratégias de aprendizagem e a ansiedade de alunos do ensino fundamental.

\section{Método}

\section{Participantes}

Os participantes eram alunos da $2^{\mathrm{a}}(n=38), 4^{\mathrm{a}}(n=40)$, $6^{\mathrm{a}}(n=40)$ e $8^{\mathrm{a}}(n=37)$ séries do ensino fundamental, compreendendo uma faixa etária de 6 a 18 anos, aproximadamente, de nível socioeconômico baixo, de uma escola da rede pública de Campinas. A amostra total contou com a participação de 155 alunos. Os alunos foram selecionados aleatoriamente de suas classes tendo por base a lista de chamada da escola. Tentou-se um equilíbrio quanto ao gênero, fato que não foi possível na $2^{\mathrm{a}}$ e na $8^{\mathrm{a}}$ série, por só haver uma turma de cada série.

\section{Instrumentos}

Os dados sobre as estratégias de aprendizagem foram coletados mediante uma entrevista individual estruturada, constituída por 16 perguntas abertas e fechadas relativas às estratégias de aprendizagem que foram traduzidas e adaptadas de Self-Regulated Learning Interview Schedule (Zimmerman \& Martinez-Pons, 1986) por Boruchovitch (1995). Esse instrumento tinha como objetivo investigar o uso de estratégias de aprendizagem por parte dos alunos em situações de aprendizagem em sala de aula, estudo em casa e realização de tarefas escolares em casa. Como exemplo de questões desse instrumento, pode-se citar a seguinte: "Vamos imaginar que a sua professora esteja dando uma aula de português e ela avise que vai dar um teste sobre aquela matéria. Você tem alguma maneira que possa lhe ajudar a aprender e a lembrar o que está sendo dado na aula? Conte para mim o que é que você faz". Maiores detalhes sobre a entrevista podem ser encontrados em Costa (2000) e em Zimmerman e Martinez-Pons (1986).
Como os instrumentos dos quais as questões foram adaptadas eram originalmente escritos em inglês, Boruchovitch (1995), num primeiro momento, traduziu-os e adaptou-os do inglês para o português e, num segundo momento, solicitou que um outro pesquisador brasileiro que morou num país de língua inglesa fizesse a versão dos instrumentos para o inglês (back translation). Acredita-se que as traduções e adaptações realizadas do instrumento empregado tenham permitido a mensuração das variáveis estudadas de forma válida e confiável.

O inventário de ansiedade traço e estado/forma infantil (IDATE) foi utilizado como instrumento para a coleta dos dados relativos a ansiedade. Esse inventário foi traduzido e adaptado por Biaggio (1983) para a realidade nacional; dessa forma, as tabelas normativas são brasileiras. A escala de ansiedade estado do IDATE-C consiste de 20 afirmações que pedem à criança que indique como se sente em um determinado momento no tempo. A escala ansiedade traço do IDATE-C também consiste de 20 itens, mas nessa escala os sujeitos devem responder como geralmente se sentem. Essa escala foi planejada para ser auto-administrável, não tendo limite de tempo. Pode ser aplicada individualmente ou em grupo. O tempo médio para a completa realização das duas escalas é de 20 minutos. No presente estudo, essa escala foi aplicada individualmente, bem como foi escolhida como instrumento, dada a inexistência de uma escala brasileira validada distinguindo a ansiedade nos aspectos preocupação e emotividade, aspectos mais destacados no referencial teórico do trabalho realizado.

\section{Procedimentos para coleta de dados}

Inicialmente foi feito contato com a Secretaria de Educação de Campinas para a obtenção de informações sobre as escolas municipais que apresentavam maiores índices de problemas de aprendizagem. Com base nas informações obtidas foram contatados os diretores de duas escolas para a apresentação dos objetivos da pesquisa e consulta sobre seu interesse em participar do estudo. Uma escola foi escolhida em virtude do interesse e da disponibilidade, enquanto a outra foi mantida como escola reserva.

Dessa forma, a coleta de dados foi realizada em uma escola pública situada na periferia de Barão Geraldo. Além dos motivos já apontados, essa instituição foi escolhida também pelo fato de atender crianças de baixo nível socioeconômico. A diretora e os professores demonstraram bastante disponibilidade para participar e concordaram em liberar os alunos durante as aulas para a realização das entrevistas. Os alunos mostraram bastante interesse em participar e aqueles estudantes de outras séries que não foram entrevistados verbalizaram estar decepcionados por não poderem tomar parte na entrevista. 
Foi explicado à diretora e à coordenadora pedagógica da escola como seria realizada a coleta de dados. Assegurou-se ainda que o nome da escola não seria mencionado e que as entrevistas seriam realizadas de modo a interferir o mínimo possível na rotina da escola.

As entrevistas foram realizadas no pátio da escola ou na sala dos professores, quando desocupada. Os dois lugares eram iluminados, com mesas e cadeiras disponíveis, e em geral silenciosos. O horário do recreio era respeitado e os alunos não eram chamados. Os participantes eram buscados individualmente nas salas de aula pela pesquisadora. Primeiramente, o aluno e a pesquisadora se apresentavam e em seguida explicava-se ao sujeito que se tratava de um trabalho com o objetivo de entender como os alunos estudam e aprendem as matérias na escola. Também era dito a ele tratar-se de uma conversa confidencial que não causaria qualquer interferência em sua vida escolar. Acredita-se que tenha sido possível o estabelecimento de um bom rapport inicial entre a pesquisadora e os alunos.

Após a apresentação e contrato, o aluno era submetido em primeiro lugar ao inventário de ansiedade IDATE (ver instrumentos). A orientação nessa hora seguiu fielmente as instruções do próprio inventário. Logo em seguida, era realizada a entrevista estruturada. Optou-se por aplicar primeiramente o inventário de ansiedade para neutralizar os efeitos da ansiedade que poderiam surgir se a entrevista (sobretudo a parte relativa às estratégias de aprendizagem) fosse aplicada primeiramente. Esses aspectos foram percebidos pela pesquisadora no estudo piloto. Os alunos responderam ao inventário de ansiedade e à entrevista num intervalo de tempo que variou de 45 a 60 minutos. Nos dias em que havia situações de avaliação, como provas e exames, as entrevistas eram sempre suspensas.

Convém ressaltar que a opção por essa medida de ansiedade é explicada pela inexistência de um instrumento validado em termos nacionais. Cabe mencionar que todo o procedimento de coleta de dados anteriormente descrito foi precedido por um estudo piloto que revelou a não necessidade de reformulação do instrumento.

\section{Procedimentos de análise de dados}

As respostas dos participantes sobre as estratégias de aprendizagem foram primeiramente estudadas por análise de conteúdo (Bardin, 1991; Berelson, 1952), utilizando-se um sistema de categorização de respostas para cada questão, com base nos sistemas já existentes na literatura (Purdie \& Hattie, 1996; Zimmerman \& Martinez-Pons, 1986). O que se pretendeu foi expandir e desenvolver um sistema de categorias que expressasse mais precisamente as idéias de alunos brasileiros e de faixas etárias pouco investigadas (Boruchovitch, 1999).
Como descrito em Boruchovitch (1993b), para aumentar a consistência da análise de dados cada categoria foi definida operacionalmente e um conjunto de regras para a classificação de uma resposta numa dada categoria foi cuidadosamente desenvolvido por Boruchovitch e Costa (ver Costa, 2000). Permitiu-se que os participantes mencionassem mais de uma estratégia para cada situação proposta. A consistência do processo de categorização foi avaliada mediante utilização de um juiz independente que analisou 32 respostas (em torno de $20 \%$ da amostra) dadas por diferentes sujeitos sobre as estratégias de aprendizagem. As respostas foram selecionadas aleatoriamente. A porcentagem de correspondência atingida foi de $90 \%$. O juiz não foi informado sobre os dados demográficos e a repetência dos estudantes durante o processo de categorização. O desempenho dos alunos no Inventário de Ansiedade foi analisado de acordo com as informações existentes no manual.

Com a finalidade de verificar possíveis relações entre o uso de estratégias de aprendizagem e a ansiedade de alunos do ensino fundamental, primeiramente analisaram-se as estratégias de aprendizagem à luz de três grandes categorias: a) utilização ou não-utilização de estratégias de aprendizagem na situação proposta; b) não-uso; utilização de estratégias de aprendizagem simples ou complexas; c) estratégias de aprendizagem cognitivas, metacognitivas ou ausência de estratégias.

O critério utilizado para classificar as estratégias de aprendizagem em simples ou complexas, bem como em cognitivas ou metacognitivas, foi baseado na taxionomia das estratégias de aprendizagem de Mckeachie, Pintrich, Lin, Smith e Sharma (1990, traduzida e adaptada por Boruchovitch, 1999), na qual os autores relacionam as estratégias de aprendizagem com o tipo de tarefas escolares (simples ou complexas) a serem realizadas pelos alunos (Anexo A). As respostas dos participantes foram lidas separadamente pela autora do estudo e por um juiz independente e classificadas de acordo com a referida taxionomia. As discrepâncias na classificação foram em torno de 5\%, sendo resolvidas por meio de discussão.

Para que se estudassem as possíveis variações no uso de estratégias de aprendizagem em função dos tipos de ansiedade (ansiedade traço/ansiedade estado) dos alunos do ensino fundamental foi utilizada a análise de variância (ANOVA). As médias da ansiedade dos alunos e os desvios-padrão nas duas escalas foram comparados com os descritos na validação feita por Biaggio (1983), e posteriormente calculados e relacionados às três grandes categorias de análise das estratégias de aprendizagem descritas anteriormente. 


\section{Resultados}

Das 16 perguntas da entrevista surgiram relações estatisticamente significativas no que diz respeito ao uso de estratégias de aprendizagem eà ansiedade somente nas situações de administração do tempo de estudo, motivação para fazer o dever de casa e organização do ambiente de estudo.

No que concerne à questão relativa a administração do tempo de estudo, os dados das Tabelas 1 e 2 parecem apontar que alunos que relataram não utilizar (ou não conhecer) nenhuma estratégia para administrar o tempo de estudo de maneira adequada mostraram uma ansiedade estado significativamente maior do que aqueles que reportaram estratégias apropriadas para a situação. Esses dados apareceram nas duas formas de análise: utilização ou nãoutilização de estratégias de aprendizagem na situação proposta $(F=5,98 ; p=0,01)$ e o não-uso, utilização de estratégias de aprendizagem simples ou complexas $(F=6,52 ; p=0,00)$.

No que concerne à motivação para fazer o dever de casa, a Tabela 3 demonstra que estudantes que mencionaram estratégias de motivação para tal tarefa têm um nível de ansiedade estado maior do que os alunos que relataram desconhecer métodos ou não utilizar estratégias de automotivação para essa situação $(F=3,95 ; p=0,04)$.

Outro aspecto abordado na entrevista foi a organização do ambiente de estudo. Foram encontradas relações significativas entre as estratégias mencionadas para organizar o ambiente de estudo e a ansiedade em três formas de análise: utilização/não-utilização de estratégias $(F=4,14 ; p=0,04)$, estratégias cognitivas e metacognitivas $(F=5,28 ; p=0,02)$, bem como estratégias simples e complexas $(F=4,79 ; p=0,03)$.

Como pode ser observado, alunos que relataram usar estratégias de estruturação do ambiente de estudo possuem um nível maior de ansiedade estado (Tabela 4) e traço (Tabela 5) do que aqueles que responderam que não organizam ou não sabem organizar o ambiente no momento de estudar.

Já a Tabela 6 mostra que sujeitos que relataram estratégias de organização do ambiente mais complexas têm uma ansiedade traço maior do que os estudantes que mencionaram não utilizar nenhuma estratégia $(F=4,79$; $p=0,03)$.

Tabela 1

Média e Desvio-padrão da Ansiedade Estado no que concerne à Utilização ou não de Estratégias de Administração do Tempo de Estudo

\begin{tabular}{lccc}
\hline Categorias de análise & $\begin{array}{c}\text { Média } \\
\text { ansiedade estado }\end{array}$ & $d p$ & $N$ \\
\hline Não utiliza & 35,28 & 5,53 & 25 \\
Utiliza & 33,19 & 3,53 & 129 \\
\hline Total & 33,53 & 3,98 & 154 \\
\hline
\end{tabular}

Tabela 2

Média e Desvio-padrão da Ansiedade Estado Relativa a não-uso, Utilização de Estratégias de Aprendizagem Simples ou Complexas para Administração do Tempo de Estudo

\begin{tabular}{lccr}
\hline Categorias de análise & $\begin{array}{c}\text { Média } \\
\text { ansiedade estado }\end{array}$ & $d p$ & $N$ \\
\hline Ausência de estratégias & 35,90 & 5,93 & 30 \\
Estratégias Simples & 32,78 & 2,83 & 59 \\
Estratégias Complexas & 33,11 & 3,62 & 38 \\
\hline Total & 33,61 & 4,16 & 127 \\
\hline
\end{tabular}

Tabela 3

Média e Desvio-padrão da Ansiedade Estado no que concerne à Utilização ou não de Estratégias de Motivação para Fazer o Dever de Casa

\begin{tabular}{lccc}
\hline Categorias de análise & $\begin{array}{c}\text { Média } \\
\text { ansiedade estado }\end{array}$ & $d p$ & $N$ \\
\hline Não utiliza & 33,01 & 3,22 & 94 \\
Utiliza & 34,30 & 4,83 & 61 \\
\hline Total & 33,52 & 3,97 & 155 \\
\hline
\end{tabular}


Tabela 4

Média e Desvio-padrão da Ansiedade Estado no que diz respeito à Utilização ou não de Estratégias de Organização do Ambiente de Estudo

\begin{tabular}{lccc}
\hline Categorias de análise & $\begin{array}{c}\text { Média } \\
\text { ansiedade estado }\end{array}$ & $d p$ & $N$ \\
\hline Não utiliza & 30,00 & 1,58 & 5 \\
Utiliza & 33,66 & 3,99 & 148 \\
Total & 33,54 & 3,99 & 153 \\
\hline
\end{tabular}

Tabela 5

Média e Desvio-padrão da Ansiedade Traço no que diz respeito ao uso ou não de Estratégias de Organização do Ambiente de Estudo

\begin{tabular}{lccc}
\hline Categorias de análise & $\begin{array}{c}\text { Média } \\
\text { ansiedade estado }\end{array}$ & $d p$ & $N$ \\
\hline Ausência & 38,18 & 5,63 & 17 \\
Estratégias metacognitivas & 41,94 & 6,45 & 138 \\
Total & 41,53 & 6,46 & 155 \\
\hline
\end{tabular}

Tabela 6

Média e Desvio-padrão da Ansiedade Traço Relacionada à Ausência on ao uso de Estratégias Complexas de Organização do Ambiente de Estudo

\begin{tabular}{lccc}
\hline Categorias de análise & $\begin{array}{c}\text { Média } \\
\text { ansiedade traço }\end{array}$ & $d p$ & $N$ \\
\hline Ausência & 38,53 & 6,27 & 19 \\
Estratégias metacognitivas & 41,95 & 6,40 & 136 \\
Total & 41,53 & 6,46 & 155 \\
\hline
\end{tabular}

\section{Discussão}

Constatar que a ansiedade se relaciona com o uso e o tipo de estratégias de aprendizagem dos alunos, como aponta a literatura da área, foi um dado parcialmente confirmado pelo presente estudo (Benjamin \& cols., 1981; NavehBenjamin, 1991; Boruchovitch \& Costa, 2001; Tobias, 1980, 1985; Wigfield \& Eccles, 1989). Mais precisamente, os resultados da presente pesquisa revelaram que alunos que parecem não ter um repertório de estratégias de aprendizagem (ausência de comportamento estratégico) possuem uma tendência a experimentar maiores níveis de ansiedade estado do que alunos que mencionaram estratégias para as situações propostas. Observou-se também que estudantes que relataram não utilizar ou não conhecer estratégias para administrar o tempo de estudo, bem como estudantes que mencionaram utilizar estratégias de automotivação e de estruturação do ambiente de estudo, apresentaram um maior nível de ansiedade (traço e estado).

Todavia, contrariando as expectativas, não foram encontradas relações significativas entre o uso de estratégias de aprendizagem e a ansiedade traço ou estado dos participantes para a maioria das situações de aprendizagem e estudo propostas pela entrevista. Aventa-se aqui a hipótese de que a medida de ansiedade (traço e estado) utilizada neste estudo pode não ter sido sensível o suficiente para mensurar de forma precisa essa relação. Seria relevante que futuros estudos examinassem essa relação valendo-se de um instrumento que captasse melhor os componentes cognitivos e emotivos da ansiedade sugeridos por uma concepção de ansiedade mais relativa à psicologia cognitiva do processamento da informação. Uma outra possível explicação para essa ausência de relação pode residir no fato de que, apesar de a alta ansiedade ser considerada pela literatura da área (Monteiro, 1980; Rocha, 1976; Tobias, 1980, 1985; Wigfield \& Eccles, 1989) como um fator que interfere na aprendizagem, a maioria das pesquisas realizadas sobre essa temática foram específicas à situação de ansiedade em relação a provas e a entrevista utilizada neste trabalho lidou com situações diversificadas de aprendizagem e estudo.

Foi interessante notar que, embora as investigações realizadas sobre a ansiedade e o desempenho escolar demonstrem que alunos com alta ansiedade possuem hábitos de estudo e estratégias de aprendizagem deficientes na preparação e realização de provas, os dados da presente pesquisa apontaram para um maior nível de ansiedade 
associado a um conhecimento deficiente sobre estratégias de administração do tempo de estudo visando somente à preparação para uma prova. Ao contrário do que era de esperar, não foram encontradas relações significativas entre a ansiedade e estratégias de realização de provas dos participantes. As possíveis relações entre estratégias de preparação, realização de provas e a ansiedade precisam certamente ser mais bem examinadas por outros estudos.

As pesquisas desenvolvidas sobre ansiedade na escola defendem que o melhor desempenho é normalmente encontrado em alunos com bons hábitos de estudo, estratégias adequadas para a realização de provas e uma ansiedade moderada (Tobias, 1980, 1985; Wigfield \& Eccles, 1989). Contudo, uma parte dos resultados do presente estudo mostrou que alunos que mencionaram usar estratégias de aprendizagem mais complexas para organizar o ambiente e para motivar-se possuíam níveis de ansiedade traço e estado ligeiramente mais elevados do que os demais. Esse achado não se assemelha aos resultados encontrados em outras pesquisas realizadas sobre o tema. Levanta-se aqui a hipótese de que talvez um maior nível de ansiedade traço e estado esteja propiciando uma maior motivação e promovendo uma melhor disposição para agir no sentido de estruturar o ambiente de forma mais apropriada.

De fato, tal como destacam alguns autores (Rocha, 1976; Sogunro, 1998), os dados do presente estudo parecem indicar que a ansiedade, nos seus diferentes níveis, tanto pode favorecer como interferir no uso adequado de estratégias de aprendizagem. Todavia, faz-se necessária a realização de outras investigações que explorem mais detalhadamente as prováveis associações entre o conhecimento e o uso de estratégias de aprendizagem e a ansiedade de alunos, já que não se constituiu num objetivo central do presente estudo fazer uma relação entre os diferentes níveis de ansiedade (baixa, média e alta) e os tipos de estratégias de aprendizagem mencionados pelos participantes em situações diversificadas de estudo e aprendizagem.

Cabe destacar que, tendo em vista a escassez de pesquisas nacionais específicas sobre essa temática, os resultados desta pesquisa foram comparados somente com a literatura internacional que, por sua vez, tem se caracterizado por estudos predominantemente voltados para a relação entre estratégias de aprendizagem e a ansiedade circunscrita a situações de provas ou avaliações.

Tendo em vista o reconhecimento cada vez maior da interferência de variáveis afetivas no rendimento escolar, é essencial que futuras pesquisas, principalmente em nível nacional, sejam realizadas no sentido de gerar um conhecimento a respeito da relação entre estratégias de aprendizagem e ansiedade. Acrescente-se a isso que a maioria dos estudos acerca dessa temática tem sido de natureza descritivo-correlacional, baseandose predominantemente em situações hipotéticas e em relatos de alunos, e não em situações reais. Pesquisas voltadas à análise da relação entre o uso de estratégias de aprendizagem e o sentimento de ansiedade em situações reais devem ser conduzidas.

\section{Considerações Finais}

Estudos recentes revelam que a intervenção em estratégias de aprendizagem auxilia o desempenho de alunos com alta ansiedade. Ao usarem as estratégias de aprendizagem, a representação cognitiva e as demandas da tarefa são organizadas de tal forma que acabam por reduzir a capacidade exigida pelo conteúdo a ser aprendido. Alunos com bons hábitos de estudo e que sabem utilizar estratégias de forma eficiente em sua preparação para as provas se beneficiarão de técnicas para reduzir a ansiedade. Estudantes com estratégias ou hábitos de estudo deficientes para a realização de testes precisam de uma intervenção que associe as estratégias de aprendizagem a tratamentos para reduzir a ansiedade (Kirkland \& Hollandsworth, 1980).

Como descrito em Boruchovitch e Costa (2001), os professores precisam aprender a lidar e saber como prevenir a ansiedade de alunos no dia-a-dia da sala de aula, ajudando-os a exercer um controle maior sobre ela, diminuindo seus efeitos negativos para a aprendizagem e para o rendimento acadêmico.

Os professores podem contribuir muito para a redução da ansiedade em sala de aula mediante certos procedimentos, como: prover os alunos com instruções sobre formas adequadas de estudo; possibilitar oportunidades para que alunos ansiosos possam falar em pequenos grupos ou responder a perguntas com respostas curtas (como sim ou não); usar atividades que envolvam aprendizagem cooperativa; esclarecer o objetivo das provas; evitar pressões de tempo nas situações de exames; determinar um espaço de tempo que assegure que todos os alunos consigam completar a prova; variar os tipos de avaliação, entre outros (Bzuneck, 1991; Bzuneck \& Silva, 1989; La Rosa, 1998; Monteiro, 1980; Naveh-Benjamin, 1991; Rocha, 1976; Sogunro, 1998; Tobias, 1980, 1985; Wigfield \& Eccles, 1989).

Pais, por sua vez, precisam ser também orientados no sentido de ajudarem na aprendizagem de seus filhos, não só durante as séries iniciais, mas em todo o período de escolarização. A importância de estruturar o ambiente físico e social no momento de estudar, a identificação dos aspectos que podem interferir ou dar um suporte positivo ao estudo, o desenvolvimento da consciência de que mais importante do que o tempo gasto para estudar é a forma como se estuda, são, sem dúvida, pontos importantes no controle da ansiedade, por parte do aluno, nos quais a parceria família-escola tornase fundamental (Dembo, 2000).

Se os estudantes tivessem a oportunidade de usar as estratégias de aprendizagem desde cedo, no transcorrer da vida acadêmica seu repertório de estratégias de aprendizagem tornar-se-ia mais complexo e flexível, possibilitando um 
melhor controle das variáveis emocionais, como a ansiedade, e um melhor desempenho acadêmico (Costa, 2000). É necessário propiciar um ensino mais efetivo, contemplando a instrução em estratégias de aprendizagem de modo a favorecer o controle da ansiedade em sala de aula, o desenvolvimento da motivação positiva para a aprendizagem, bem como possibilitar a passagem gradual da regulação da aprendizagem realizada pelo educador para o controle pessoal exercido pelo aluno (Bronson, 2000; Dembo, 2000; Hacker, Dunlosky \& Graesser, 1998; Zimmerman, 1998).

\section{Referências}

Ajuriaguerra, J. (1976). Manual de psiquiatria infantil. Rio de Janeiro: Masson do Brasil. Bardin, L. (1991). Análise de conteúdo. Lisboa, Portugal: Edições 70.

Benjamim, M., McKeachie, W. J., Lin, Y. G. \& Holinger, D. P. (1981). Test anxiety: Deficits in information processing. Journal of Educational Psychology, 73, 816-824.

Berelson, B. (1952). Content analysis in communications researches. Glencoe: Free Press.

Biaggio, A. M. B. (1983). Inventário de ansiedade traço-estado (IDATE-C). Rio de Janeiro: CEPA.

Biaggio, A. M. B. (1998). Ansiedade, raiva e depressão na concepção de C. D. Spielberger. Revista de Psiquiatria Clinica, 25, 291-293.

Boruchovitch, E. (1993a). A psicologia cognitiva e a metacognição: Novas perspectivas para o fracasso escolar brasileiro. Tecnologia Educacional, 22, 110-111.

Boruchovitch, E. (1993b). Health and illness-realted cognitions among Brarilian students: A cross-cultural contribution. Tese de Doutorado não-publicada, School of Education, University of Southern California. Los Angeles/CA, EUA.

Boruchovitch, E. (1995). A identificacão e o estudo das variáveis associadas ao fracasso escolar brasileiro. Projeto de pesquisa realizado na qualidade de bolsista de recémdoutor do CNPq. Faculdade de Educação, Departamento de Psicologia Educacional, Universidade de Campinas.

Boruchovitch, E. (1999). Estratégias de aprendizagem e desempenho escolar: Considerações para a prática educacional. Psicologia: Reflexão e Crítica, 12, 361-376.

Boruchovitch, E. \& Costa, E. (2001). O impacto da ansiedade no rendimento escolar e na motivação de alunos. Em E. Boruchovitch \& J. A. Bzuneck (Orgs.), Motivação do aluno: Contribuições da psicologia contemporânea (pp. 134147). Petrópolis, RJ: Vozes.

Bronson, M. B. (2000). Self regulation in early childhood. New York, London: The Guilford press.

Brown, A. L. (1997). Transforming school into communities of thinking and learning about serious matters. American Psychologist, 52, 399-413.

Bzuneck, J. A. (1991, maio). Ansiedade e desempenho numa prova de matemática: Um estudo com adolescentes. Trabalho apresentado no II Simpósio interdisciplinar de estudos cognitivos, Laboratório de Estudos Cognitivos da Universidade Estadual de Londrina, Londrina, Paraná.

Bzuneck, J. A. \& Silva, R. (1989). O problema da ansiedade nas provas; perspectivas contemporâneas. Semina,10, 190-195.

Corno, L. \& Mandinach, E. B. (1983). The role of cognitive engagement in classroom learning and motivation. Educational Psycologist, 18, 88-108.

Costa, E. R. (2000). As estratégias de aprendizagem e a ansiedade de alunos do ensino fundamental: Implicações para a prática educacional. Dissertação de Mestrado em Educação não-publicada, Curso de Pós-Graduação em Psicologia Educacional, Universidade Estadual de Campinas. Campinas, SP.
Da Silva, A. L. \& De Sá, L. (1997). Saber estudar e estudarpara saber. Cidade do Porto, Portugal: Porto.

Dembo, M. H. (1994). Applying educational psychology (5 $5^{\text {th }}$ ed). New York: Longman.

Dembo, M. (2000). Motivation and learning strategies for college success. New Jersey: Lawrence Erlbaum.

Eysenck, M. W. (1992). Anxiety the cognitive perspective. Hillsdale, USA: Lawrence Erlbaum/Royal Hollowly and Bedford New College, University of London.

Flavell, J. H., Miller, P. H. \& Miller, S. A (1999). Desenvolvimento cognitivo (3 a ed). Porto Alegre: Artes Médicas.

Freud, S. (1894). Obras completas (Vol. 2). Rio de Janeiro: Imago.

Hacker, D. J., Dunlosky, J. \& Graesser, A. C. (1998). Metacognition in educational theory and practice. New Jersey: Lawrence Erlbaum.

Kirkland, K. \& Hollandsworth, J. G. Jr. (1980). Effective test taking: Skills acquisition versus anxiety reduction techniques. Journal of Consulting and Clinical Psychology, 48, 431-439.

La Rosa, J. (1998). Ansiedade, sexo, nível sócio-econômico e ordem de nascimento. Psicologia: Reflexão e Crítica, 11, 59-70.

May, R. (1980). Significado da ansiedade. Rio de Janeiro: Zahar.

Monteiro, M. N. (1980). Um estudo da ansiedade e suas implicações no desempenho acadêmico. Dissertação de Mestrado não-publicada, Curso de Pós Graduação em Psicologia Clínica, Pontifícia Universidade Católica do Rio de Janeiro. Rio de Janeiro, RJ.

Morris, L. W., Davis, M. A. \& Hutchings, C. H. (1981). Cognitive and emotional components of anxiety Literature review and a revised worry emotionality scale. Journal of Educational Psychology, 73, 541-555.

Naveh-Benjamin, M. (1991). A comparison of training programs intended for different types of test-anxious students: Futher support for an informationprocessing model. Journal of. Educational Psychology, 83, 134-139.

Pessoti, I. (1977). Estudo sobre o conceito de ansiedade. Tese de Livre Docência da Universidade de São Paulo. Ribeirão Preto, SP.

Pozo, J. I. (1995). Estratégias de aprendizagem. Em C. Coll, J. Palácios \& A. Marchesi (Orgs.), Desenvolvimento psicológico e educação: Psicologia da educação (Vol. 2, pp. 176-197). Porto Alegre: Artes Médicas.

Purdie, N. \& Hattie, J. (1996). Cultural differences in the use of strategy for selfregulated learning. American Educational Research Journal, 33, 845-871.

Rocha, I. (1976). Estudos de ansiedade escolar: Avaliação de situações positivas e negativas, ansiedade e nivel de realização. Dissertação de Mestrado não-publicada, Curso de Pós-Graduação em Psicologia, Universidade de São Paulo. São Paulo, SP.

Rosamilha, N. (1971). Psicologia da ansiedade infantil. São Paulo: Pioneira.

Sogunro, O. (1998). Impact of evaluation anxiety on adult learning. Journal of Research and Development in Education, 31, 109-120.

Tobias, S. (1980). Anxiety and instruction. Em I. G. Sarason (Org.), Test anxiety: Theory, research and applications (pp. 289-309). Hillsdale, NJ: Erlbaum.

Tobias, S. (1985). Test anxiety: Interference, defective skills and cognitive capacity. Educational Psychologist, 20, 135-142.

Wigfeld, A. \& Eccles, J. S. (1989). Test anxiety in elementary and secondary school students. Educational Psychologist, 24, 159-183.

Woolfolk, A. (2000). Psicologia da educação. Porto Alegre: Artes Médicas.

Zimmerman, B. J. (1986). Academic studying and the development of personal skill: A self-regulatory perspective. Educational Psychology, 33(2/3), 73-86.

Zimmerman, B. J. (1998). Becoming a self-regulated learner: which are the key subprocesses? Contemporary Educational Psychology, 11, 307-313.

Zimmerman, B. J. \& Martinez-Pons, M. (1986). Development of a structured interview for assessing student use of self-regulated learning strategies. American Educational Research Journal, 23, 614-628.

Recebido: 08/01/2003

$1^{a}$ Revisão: 20/03/2003

Última Revisão: 07/05/2003

Aceite Final: 27/05/2003

Sobre as autoras

Elis Regina da Costa é Doutoranda do Programa de Pós-Graduação da UNICAMP, Faculdade de Educação, Departamento de Psicologia Educacional. Trabalho adaptado da dissertação de mestrado da primeira autora, realizada sob orientação da segunda autora. Projeto financiado pela FAPESP (Proc. N 98/10615-0).

Evely Boruchovitch é Professora Assistente-Doutor da UNICAMP-Faculdade de Educação, Departamento de Psicologia Educacional, UNICAMP. Membro do Grupo de Estudos e Pesquisas em Psicopedagogia (GEPESP) UNICAMP. 


\title{
ANEXO A \\ Taxioma das Estratégias de Aprendizagem
}

\author{
Estratégias Cognitivas
}

\begin{tabular}{lll}
\hline Estratégias & Tarefas Simples & Tarefas Complexas \\
\hline Ensaio & Repetir & $\begin{array}{l}\text { Copiar o material } \\
\text { Anotar na íntegra } \\
\text { Sublinhar }\end{array}$ \\
& & Parafrasear \\
& R. Mnemônicos & $\begin{array}{l}\text { Resumir } \\
\text { Anotar } \\
\text { Claboração analogias }\end{array}$ \\
& & Criar \\
& R. Minemônicos & Selecionar idéias \\
& Roteiros, Mapas
\end{tabular}

Estratégias Metacognitivas

\begin{tabular}{ll}
\hline Estratégias & Todas as Tarefas \\
\hline Planejamento & Estabelecer metas \\
Monitoramento & Auto-testagem \\
& Atenção \\
& Compreensão \\
& Uso de estratégias \\
& Ajustar velocidade \\
Regulação & Reler \\
& Rever \\
& Uso de estratégias \\
& Ajustar ambiente \\
\end{tabular}

Traduzido e adaptado por Boruchovitch (1999) de Mckeachie, Pintrich, Lin, Smith e Sharma (1990, citado em Dembo, 1994). 